\title{
Anterior teeth rehabilitation with direct resin composite veneer using multiple layering technique: A case report
}

\author{
Muhammad Nur Izham Khairuddin, Pengiran Muhammad Badi'uzzaman Awang
} Iskanderdzulkarnein, Mohd Haikal Mohd Halil ${ }^{*}$

Department of Restorative Dentistry, Kulliyyah of Dentistry, IIUM Kuantan Campus

\begin{abstract}
Cosmetic veneer has become a popular option for patients seeking to improve aesthetic in their smile. The procedure can be done through a direct or indirect technique which differs in term of material used and laboratory involvement. Despite producing a better aesthetic result than its direct technique counterpart, high-cost treatment, multiple visit requirement and invasive nature of tooth preparation are proven to be a stumbling block to the patient in some cases to choose indirect technique. Therefore, direct composite veneer technique can be offered due to its low costing and conservative concept which advocates more tooth structure preservation. This case report is about direct composite veneer for anterior teeth rehabilitation and its simple and conservative protocol.
\end{abstract}

Keywords: veneer, aesthetic, composite
Received:

13 August 2020

Revised:

7 December 2020

Accepted:

14 December 2020

Published Online:

28 February 2021

\section{How to cite this article:}

Khairuddin, M. N. I. ., Awang Iskanderdzulkarnein, P. M. B. ., \& Mohd Halil , M. H. . Anterior teeth rehabilitation with direct resin composite veneer using multiple layering technique: A case report. IIUM Journal of Orofacial and Health Sciences, 2(1). 56-61. https://doi.org/10.31436/ijohs.v2i $\underline{1.38}$ Article DOI:

https://doi.org/10.31436/ijohs.v2i1. $\underline{38}$

Corresponding author:

Address:

Department of Restorative Dentistry, Kulliyyah of Dentistry, IIUM Kuantan Campus.

Telephone: $+6019-2816007$

Email address:

drhaikal@iium.edu.my

\section{Introduction}

Aesthetic dentistry as defined by Mosby's Dental Dictionary as "the skills and techniques used to improve the art and symmetry of the teeth and face to enhance the appearance as well as the function of the teeth, oral cavity and face" (Elsevier, 2014). Important aesthetic elements like shape or form of tooth, symmetry and proportionality, position and alignment, surface texture, colour and translucency play an important role in producing optimal aesthetic smile in addition to proper function and phonetics (Heymann and Ritter, 2019). Loss or absence of one or more of these elements will possibly from dental caries, trauma, or malformations either congenitally or acquired will compromise the general appearance of a smile as a whole which can affect a person's self-confidence and motivations.

To rehabilitate and rejuvenate affected smile due to loss of key aesthetic smile elements, there are several treatment options available depending on the clinical considerations, for example, cosmetic contouring, direct composite restoration, dental crown, veneer and micro-abrasion. One of the most common indicated treatment options to restore the aesthetic features of a tooth is dental crowns (Hickel et al., 2004). In dental crown treatment, remaining tooth structure is prepared to receive the crown itself which include the 
removal of healthy dental hard tissue to accommodate the physical demand of the restoration. The extensive removal of tooth structure carries the risk of damage to the pulp for vital tooth and surrounding tissue like gingiva (Aristidis and Dimitra, 2002).

Another treatment option that involves less tooth structure removal and achieves a comparable aesthetic result to the conventional dental crown is veneers. Veneer involves the application of a layer of tooth-coloured material on tooth surface particularly labial or buccal surface, to restore localised or generalised defects and intrinsic discolourations (Heymann and Ritter, 2019). Depending on cases, a veneer can be done either through a direct or indirect technique which differs in term of material selection and dental laboratory involvement. A direct veneer technique can be done by application of resin composite on minimally or non-prepared tooth surface and completed chair-side in one appointment. Direct veneer technique or direct composite veneer (DCV) can be considered over indirect technique when the patients are time or financially limited, young patients or a single discoloured tooth (Heymann and Ritter, 2019). There are also some of the reported indications for DCV which include tooth discolouration, rotated teeth, congenital or acquired malformations, diastema, discoloured restorations, palatally positioned teeth, absence of lateral incisors, abrasions and erosions (Hickel et al., 2004).

This case report describes the direct composite veneer technique in aesthetic rehabilitation of anterior teeth in a young adult patient related to the slanted position of teeth, overhanging restorations and secondary caries under old restorations which affect the general aesthetic of a smile.

\section{Case report}

A 25-year-old healthy Malay male patient came to dental polyclinic at Kulliyyah of Dentistry, International Islamic University Malaysia (IIUM) in January 2020 with concern regarding the appearance of his upper anterior teeth. His main concerns were the mismatched colour of previous tooth restoration with the natural tooth and unaesthetic appearance of the incisal edge of the upper incisors. According to the patient's dental history, the teeth were restored due to caries with no complaints or complications afterwards. The patient seeks the replacement of less-than-desirable older restorations and fixes the appearance of the incisors with regard to the overall shape and form of the incisors.

Upon clinical examinations, the existing restoration appears to be discoloured and there is secondary caries detected at the mesial of left maxillary central incisor and over-hanging restoration on the mesial of the right maxillary central incisor. The incisal edge of both maxillary central incisors was also slanted unfavourably to the patient's aesthetic needs (Figure 1). Pulp sensibility and periodontal probing were also done to assess the pulp and periodontal status of the tooth in which all the tooth that need to be restored responded normally to the test and free of periodontal problem. Occlusion between both upper and lower dentition were also assessed to ensure harmonious inter-arch occlusion relationship (Figure 2). The remaining tooth structures were adequate for both teeth to receive dental veneers but due to the limited budget and time constraint, the patient agrees to the DCV method for both teeth instead of indirect veneer technique. The tooth will be restored directly using resin composite by removing previous restorations with minimal preparations for the veneer. Incorporation of proper incisal edges also will be done along with an attempt to recreate natural anatomical features as close as possible.

A diagnostic wax-up was made on the study cast to give an overview of the expected outcomes of the restorations to the patient (Figure 3). Silicone index was also made from the wax-up which include from upper right canine to the left contralateral side to serve as guide in placement of palatal shell. The tooth shade was determined before the commencement of treatment using VITA classical A1-D4® shade guide (VITA Zahnfabrik, Bad Säckingen, Germany) and 
matched with the custom resin composite shade guide with Ceram $\cdot X ®$ Duo i-Shade (Dentsply Sirona, Konstanz, Germany). In this case, based on the custom resin composite shade guide, shade E2 was chosen as enamel shade while shade D2 for the dentinal shade of the restoration.

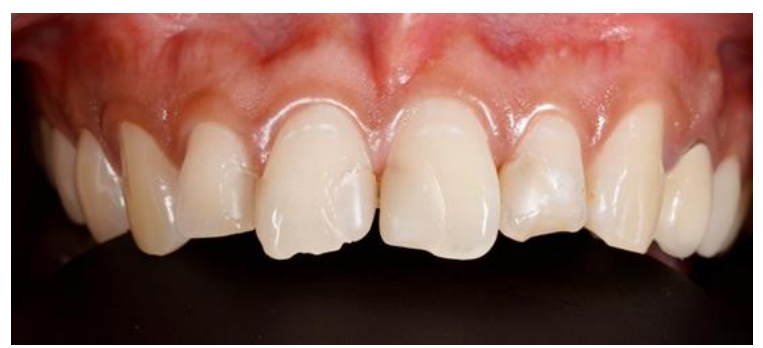

Figure 1. Pre-operative view. Slanted incisal angles of tooth 11 and 21, a visible shadow also noted at mesial of 21 indicated secondary caries under the existing restoration.

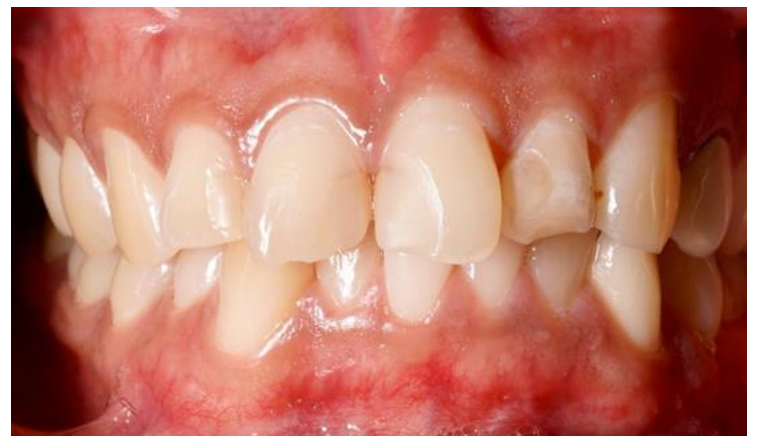

Figure 2. Pre-operative view. Inter-ach relationship of both upper and lower anterior dentition.

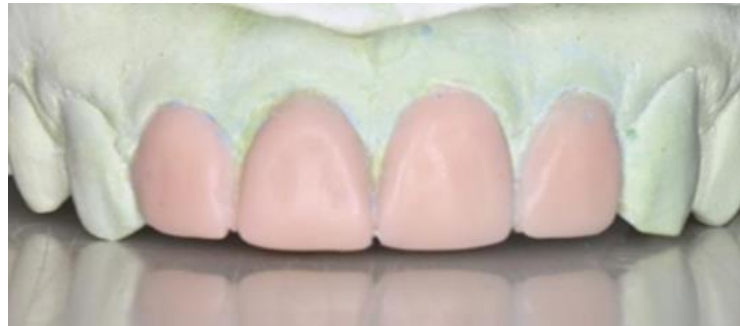

Figure 3. Diagnostic cast with wax up on tooth 13 until 23. Putty matrix was prepared based on this diagnostic wax-up including from tooth 13 until 23.

Following maxillary infiltration on the respective tooth was done, a rubber dam is placed using multiple isolations from right maxillary canine to the left maxillary canine. Removal of previous restoration and carious tissue was done until caries-free is achieved on all of the teeth with round shaped diamond bur. Affected dentine present at the prepared tooth is left unprepared and covered with glass ionomer cement as a liner (Figure 4). Minimal preparation was made on the labial surface to include beveling and conservative labial reduction of $0.3 \mathrm{~mm}$ using diamond chamfer bur. The selective etching was done on the enamel surfaces with $37 \%$ phosphoric acid (Eco-Etch ${ }^{\circledR}$ gel,
Ivoclar Vivadent, Liechtenstein) for 15 seconds and rinsed and air-dried. Bonding agent (Prime\&Bond $\AA$ universal, Dentsply Sirona, Konstanz, Germany) was applied thoroughly and light-cured for 20 seconds.

For resin composite placement (ceram.x duo®, Dentsply Sirona, Konstanz, Germany), palatal shell and the proximal wall was first established with enamel shade of E2 guided using silicone index constructed beforehand (Figure 5 \& Figure 6). Subsequently, resin composite (ceram.x duo®, Dentsply Sirona, Konstanz, Germany) with shade D2 is used for dentine layering which was done incrementally and light-cured for 20 seconds 
on each layer (Figure 7). The final layer of the labial surface is restored with resin composite enamel shade of E2 and light cured for 20 seconds before removal of the rubber dam. Each layering and contouring done using flat end plastic instrument along with silicone sculptor to ensure proper adaptation and smooth transition between restoration and tooth structure.

Finishing of the restoration was done using fine needle diamond bur and abrasive disk (Super Snap, Shofu Inc, Kyoto, Japan) to contour the surface anatomy. Final finishing is then completed using Enhance $\AA$ (Dentsply Sirona, Konstanz, Germany) system with polishing paste (Prisma-Gloss ${ }^{\circledR}$, Dentsply Sirona, Konstanz, Germany). The restoration then polished with $\mathrm{PoGo}^{\mathrm{TM}}$ polishers (Dentsply Sirona, Konstanz, Germany). Each tooth is restored using the same protocol simulating to the adjacent tooth (Figure 8). After one week, the patient was recalled for review and there was no post-operative sensitivity and aesthetic or functional problem (Figure 9).

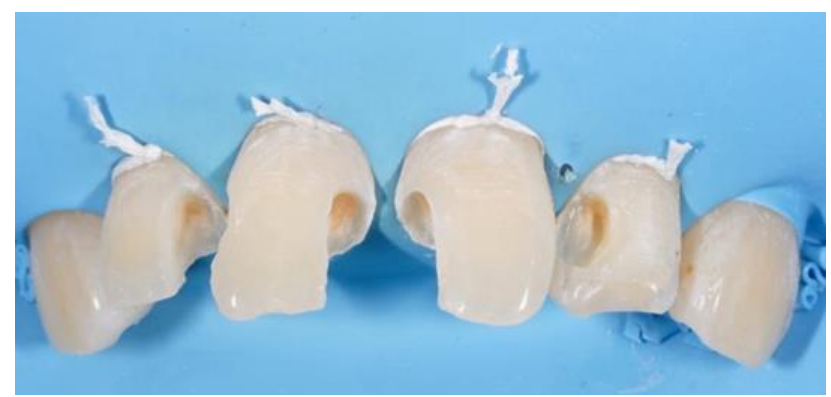

Figure 4. Rubber dam placement and removal of previous restoration and carious tissue. Notice the affected dentine was left unprepared and will be covered with liner.

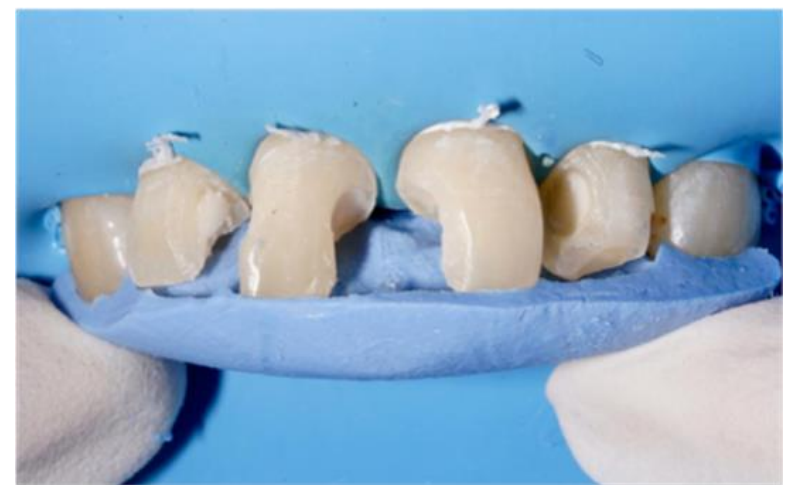

Figure 5. Silicone index fabricated prior to the treatment was used as guide to build up palatal shell and proximal border.

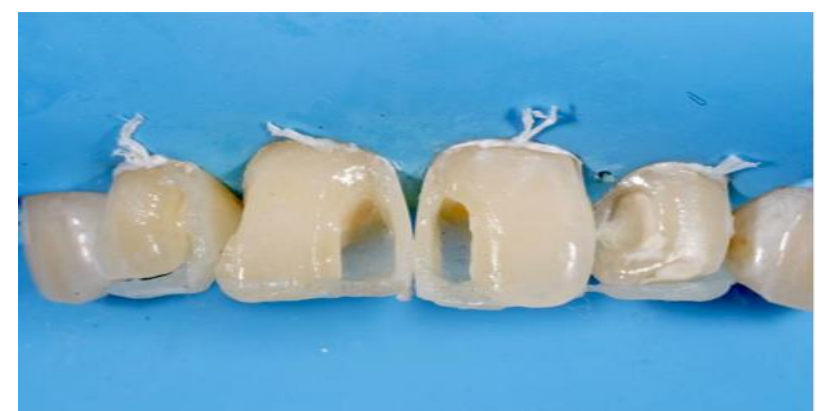

Figure 6. Both palatal shell and proximal wall were built up using ceram.x® duo shade E2, to represent the enamel layer. 


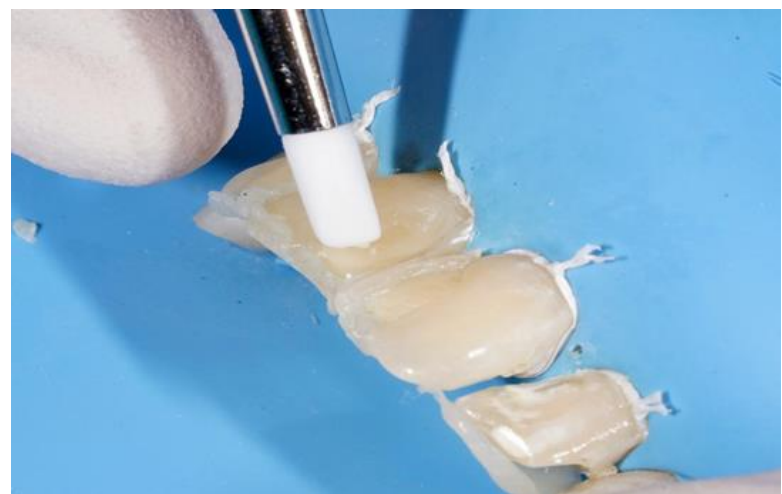

Figure 7. Application and layering of dentinal layer using ceram.x ${ }^{\circledR}$ duo shade $D 2$. The procedure was aided by using silicone sculpture brush to ensure proper adaptation and smooth transition for each layering.

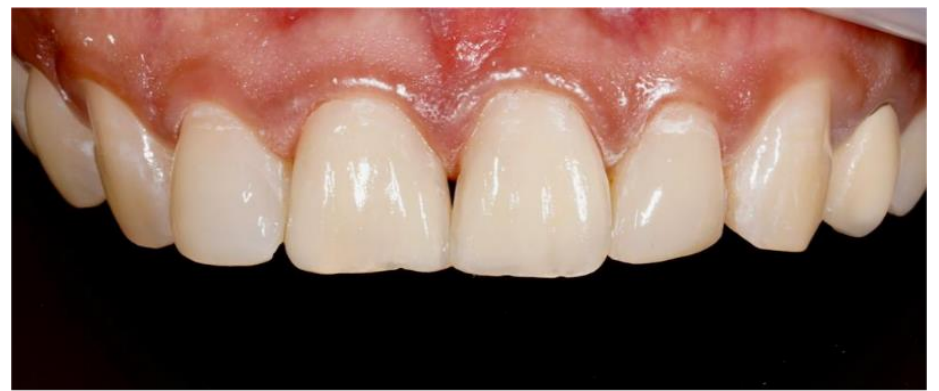

Figure 8. Post-operative appearance of the restoration

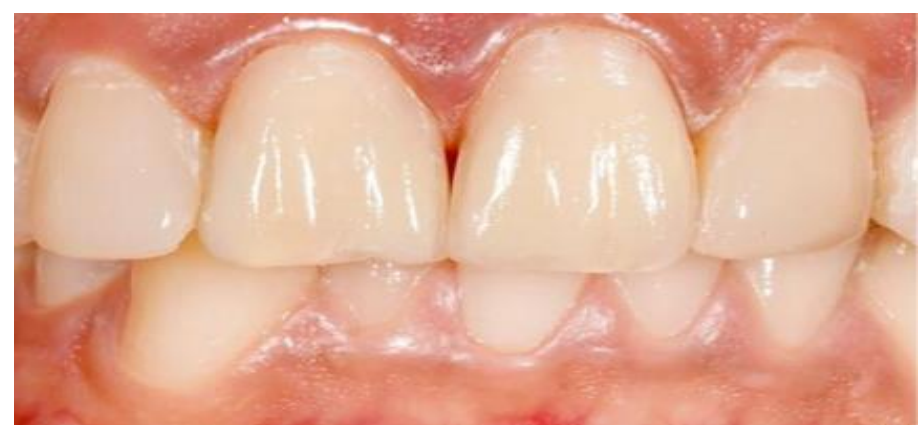

Figure 9. The restoration at one-week recall shows the optimal blending between restoration and tooth structure.

\section{Discussion}

This case report describes the use of resin composite in direct composite veneer technique in rehabilitating the appearance of less-than-desired upper anterior teeth particularly the incisors. The need for affordable and less hassle aesthetic dentistry is gaining popularity in the practice of modern dentistry and direct composite veneer are amongst the treatment of choice offered to the patient. In this case, the patient opted for direct laminate veneer treatment using composite resin although indirect technique offers more advantages over direct technique (Heymann and Ritter, 2019).

Resin composite materials used in direct veneer technique are known to be associated with long-term discolouration, lower physical strength in term of wear and fracture resistance in indirect technique in addition to the labour-intensive procedure in one appointment for the patient. However, direct composite veneer technique offers a more conservative approach in tooth preparation, low cost in term of material used and elimination of laboratory procedure besides 
the whole procedure can be done completely chair-side in one appointment. The versatility of the resin composites itself means it can be repaired and polished intra-orally without the need to remove the existing restorations which then require more tooth structure removal. The success rate of direct composite veneer also has $89 \%$ success rate for five years as reported by Peuman et al. (1997). With the advancement in dental materials technology, more resin composites material with better handling and physical properties will be produced in the future and soon the gap between both direct and indirect veneer technique will be much closer.

Another point to ponder in this case is the use of silicone index in establishing palatal shell and the proximal wall along with mylar strip. The usage of this technique will reduce the risk of loss proximal contact point and overhung restoration as illustrated in the beginning of this case. Improper adaptation of mylar strip without fixing it with wedges and inability to appreciate proper physiologic contour of the tooth is the main contributing factor the problem stated previously. Therefore, incorporating the use of silicone index in direct composite veneer protocol should be practised whenever possible (Ammannato et al., 2017).

As for the layering technique used in this case, bilaminar layering with natural layering shading was used to utilise the CLOUD shade effect of the resin composite used (ceram.x duo®, Dentsply Sirona, Konstanz, Germany) which has enhanced chameleon blending ability. In this technique, natural tooth layers are used as model and emulated by the resin composite used with single body shade used for both dentine and enamel layering. Resin composite that emulates this concept use combination of universal shading of dentine with single opacity with ranges of chrome and different tint and translucency for the enamel shade (Dietschi and Fahl, 2016). With much simplified shading and layering, this technique reduces the armamentarium and material needed for the procedure and making it less demanding compared to much more complicated layering technique such as trilaminar and polychromatic approach.

\section{Conclusion}

From this case report, it can be concluded that the direct composite veneer technique is a viable treatment option to be offered to the patient seeking rehabilitation of anterior teeth but has certain limitations. With judicious application of resin composite and good appreciation physiologic contour and anatomy of the tooth can made attractive smile achievable with simpler clinical protocol.

\section{References}

Ammannato, R., Ferraris, F., \& Allegri, M. (2017). The "index cutback technique": a three-dimensional guided layering approach in direct class IV composite restorations. The International Journal of Esthetic Dentistry, 12(4), 450-466.

Aristidis, G. A., \& Dimitra, B. (2002). Five-year clinical performance of porcelain laminate veneers. Quintessence International, 33(3), 185189.

Dietschi, D., \& Fahl, N., Jr. (2016). Shading concepts and layering techniques to master direct anterior composite restorations: an update. British Dental Journal, 221(12), 765-771.

Elsevier, M. (2014). Mosby's Dental Dictionary - E-Book, (3rd ed.)., Elsevier Inc, 236-237.

Ritter, A. V. (2017). Sturdevant's Art \& Science of Operative Dentistry - E-Book, (7th ed)., 264-305.

Hickel, R., Heidemann, D., Staehle, H. J., Minnig, P., Wilson, N. H., German Scientific Association for Operative Dentistry, \& European Federation of Conservative Dentistry (2004). Direct composite restorations: extended use in anterior and posterior situations. Clinical Oral Investigations, 8(2), 43-44.

Peumans, M., Van Meerbeek, B., Lambrechts, P., \& Vanherle, G. (1997). The 5-year clinical performance of direct composite additions to correct tooth form and position. I. Esthetic qualities. Clinical Oral Investigations, 1(1), 12-18. 